\title{
Analysis of TCT Combined with HPV Typing Detection in Cervical Lesions Screening of Female Medical Staff in Obstetrics and Gynecology Hospital
}

\author{
Hu Min-Min* \\ Department of Preventive Health Care, Obstetrics and Gynecology Hospital of Fudan University, China \\ *Corresponding author: HU Min-Min, Department of Preventive Health Care, Obstetrics and Gynecology Hospital of \\ Fudan University, Shanghai 200011, China
}

\begin{abstract}
ARTICLE INFO
Received: 慧 March 26, 2020

Published: 慧 April 28, 2020

Citation: Hu Min-Min. Analysis of TCT Combined with HPV Typing Detection in Cervical Lesions Screening of Female Medical Staff in Obstetrics and Gynecology Hospital. Biomed J Sci \& Tech Res 27(2)2020. BJSTR. MS.ID.004482.
\end{abstract}

Keywords: HPV; High risk HPV; Female Medical Workers

\section{ABSTRACT}

Objective: This study was to analyze the physical examination and screening data of female medicalstaff in a maternity hospital in Shanghai, and to study the relationship between liquid-based cytology andhuman papillomavirus (HPV) infection.

Methods: TCT results of 1093 female medical staff in the hospital from2015 to 2017 and HPV results of 1085 cases in 2017 were collected. SurePath liquid-based cell preparation wasused for cytology and the segr-21 typing kit was used for HPV detection. The relationship between thepathological results of cervical colposcopy biopsy and HPV infection was studied by single factor and multiplefactor regression analysis.

Result: The rate of HPV infection women, who were aged 22-90 years old in thisresearch was $10.0 \%(108 / 1085)$ and increased with age. High-risk HPV infection accounted for $95.37 \%(103 / 108)$, and the major high-risk HPV infection was HPV $52,53,58$, and 16 in descending order. The 3-yearcytological positive rates were $1.5 \%, 1.6 \%$ and $4.0 \%$, respectively. Sixty-six of the HPV and/or cytologicalpositive cases underwent colposcopy biopsy, and the proportion of cytological positive, HPV positive, HPV16positive, cytological negative in 2015 and 2016 was 41.1\%, 19.1\%, $60 \%$ and $29.7 \%$, respectively. Multiplelogistic regression showed that cytology was an independent risk factor, OR 7.87 (95\%CI: 1.85-33.54).

Conclusion: The rate of HPV infection in medical staff was lower than that in the general population, but thecomposition ratio of high-risk HPV infection was very high. It was recommended that women should be carriedout HPV test regularly from sexual life, and early detection of high-risk HPV infection could prevent theoccurrence of cervical cancer. The sensitivity and specificity of cervical cytology screening were higher thanHPV screening in people with low infection rate, and the cytology screening cycle should not exceed one year.

\section{Introduction}

Cervical cancer is a malignant tumor that seriously harms women's health. According to the World HealthOrganization, about 70,000 new cases of cervical cancer were found worldwide in 2018, and more than 310,000cases of death because of cervical cancer. The incidence rate of middle-income and low-income Asian andAfrican countries was 76.2\% [1]. Human papillomavirus (HPV) infection is recognized as an important cause ofcervical cancer. At least 13 types of HPV high-risk infections may cause cancer. Among which, HPV16 and HPV18high-risk subtype infections has a possibility as high as $70 \%$ to cause cervical cancer or cervical cancerprecancerous lesions [2]. There are generally different stages before an infection of HPV could become cervicalcancer. The pathological changes of the cervical epithelium can be found by Papanicolaou test. Study shows thatthe incidence of cervical cancer has a younger trend. Since the 1950s, European and American countries haveused Papanicolaou test to perform cervical cancer screening and has greatly reduced the incidence andmortality of cervical cancer [3].

Therefore, there is significant importance and meaning for women to take HPVtest regularly, it will help detecting high-risk 
HPV infection and thus be treated timely. The cervical liquidbasedcytology method developed in the 1990s has gradually replaced the traditional Pap smear in many countries,further improving the detection rate of cervical lesions. The rise of HPV testing has led to debates about whetherHPV testing can replace cytology as a first-line screening method for cervical lesions. In the 2016 study "TheAmerican College of Obstetricians and Gynecologists. Cervical Cancer Screening and Prevention", it isrecommended that women aged $3-=65$ years should give priority to the combination of cytology and HPVscreening [4]. In recent years, studies and guidelines have proposed single HPV testing is a viable screeningmethod for cervical cancer [5]. There has always been a widespread attention and debate on the choice of theright method to detect cervical cancer and that for specific groups.

In China, a number of studies have focused on HPV infection rates [6,7] and pap smear test positive rates [8]. Medical staff is a special occupational group as they have higher awareness of HPV infection than the generalpopulation and have more medical expertise. However, staff at obstetrics and gynecology hospitals are of highriskgroup as they're expected to be exposed to HPV infection. There's relatively small infection rate data of thiscertain group of people and whether the incidence of HPV infection and cervical lesions is higher than ordinarywomen are still unclear. By improving and analyzing the screening data of the hospital's obstetricians andgynecologists, the basic data will be accumulated for the primary prevention and screening methods for highriskgroups in the future. This study analyzed the cytology and HPV characteristics of this specific group ofmedical staff from Shanghai. We also analyzed the effectiveness of various methods to prevent precancerouslesions.

\section{Materials and Methods}

\section{Research Object}

Data was collected from the cervical cancer screening data of all active and retired female medical stuff ofObstetrics and Gynecology Hospital of Fudan University from 2015 to 2017. The screening period is once peryear, with an interval of approximately 12 months. Thin prep cytology test was done during 2015-16, and HPVscreening was added in 2017. The total number of people undergoing physical examinations in 2017 hasincreased compared to the past as it includes new employees as well as employees from 2015 and 2016. Thenumber of tested samples in three years was 660,729 , and 1049

\section{Research Objects and Methods}

\section{HPV Test Method}

The 21 full-type HPV test kit from JIANGSU BIOPERFECTUS TECHNOLOGIES COMPANY, LTD was used for thetest. Thirteen types of high-risk types HPV $(16,-18,-31,-33,-35,-39,-45,-51$, $-52,-56,-58,-59$ and -68$)$, fivepotential high-risk types (26,
$-53,-66,-73$ and -82$)$, and three low-risk types $(-6,-11,-81)$ were simultaneouslydetected by type-specific primers. The classification of the three HPVs is based on IARC's carcinogenic potentialof HPV, that is, the high-risk type is carcinogenic or highly likely to be carcinogenic; the potentially high-risktype is likely to be carcinogenic; the low-risk type is non-carcinogenic [9] Intracellular single copy gene DNAtopoisomerase 3 was used as a relative quantitative internal reference gene. Both PCR system and amplificationassay is operated according to the product instructions. The result is based on the threshold set by the device.

\section{TCT (Thinprep Cytology Test) Method}

TCT was collected and maintained using a Sure Path cervical sampler and preservation solution (Becton,Dickinson and Company, Franklin Lakes, USA). After mixing, $2 \mathrm{ml}$ of the sample was taken for HPV detection,and the remaining samples were used for TCT test. The cell preparation was conducted with BD PrepStainautomatic processor and with the dyeing machine from Becton, Dickinson and Company. The procedure was

carried out according to the procedure provided by the company.The results for the TCT test is either atypical squamous cell of uncertain significance, ASC-US or positive,including ASC-US, low grade squamous intraepithelial lesion, LSIL and high grade squamous intraepitheliallesion, HSIL.

\section{Statistical Analysis}

Data analysis was conducted by using Excel 2007 and SPSS 20.0 (...software). The prescriptive analytics resultswere presented by mean, standard deviation, median and percentile. The HPV infection rate of different agegroups was compared using ChiSquared Test, the amount and percentage were presented as composition ratio(\%). $\chi 2$ test was used for univariate analysis and logistic regression was used for multivariable analysis with atest level of $\alpha=0.05$.

\section{Result}

\section{Overall Summary}

The number of medical stuff who participated in the TCT test in 2015 and 2016 was 670 and 741,respectively, while in 2017, the number of samples for TCT and HPV test were 1093 and 1085, 8 of them onlyparticipated in the TCT test. The age distribution ranged from 22 to 90 years, with an average age of 41.6 years(41.6 years \pm 14.0 years) and a median age of 37 years, with upper and lower quantile of 30 and 49 . Accordingand $4.0 \%$ respectively, and the ASC-US rate were $0.9 \%, 0.9 \%$ and $3.1 \%$ (Table 1 ). The positive and ASC-US ratein 2017 were significantly higher than that in 2015 and 2016 Pearson $\chi 2=16.03, \mathrm{P}<0.01$ ). Colposcopy andhistological biopsy were performed to 66 patients with positive TCT tests (ASC-US and above) in all three years. The results showed that 51 patients $(77.3 \%)$ were normal (negative for intraepithelial lesion or malignancy,NILM), 13 (19.7\%) were LSIL and 2 were HSIL. 
Table 1: 2015-2017 TCT Test Results of Female Medical Staff in a First-class Hospital at Grade 3 inShanghai.

\begin{tabular}{|c|c|c|c|c|c|}
\hline NILM (\%) & $\mathbf{2 0 1 5}$ & $\mathbf{2 0 1 6}$ & $\mathbf{2 0 1 7}$ & Pearson $\mathbf{2}$ & $\mathbf{P}$ \\
\hline & $660(98.5)$ & $729(98.4)$ & $1049(96.0)$ & 14.62 & 0.001 \\
\hline ASC-US and above(\%) & $10(1.5)$ & $12(1.6)$ & $44(4.0)$ & 14.62 & 0.001 \\
\hline ASC-US(\%) & $6(0.9)$ & $7(0.9)$ & $34(3.1)$ & 16.03 & $<0.01$ \\
\hline LSIL (\%) & $3(0.4)$ & $3(0.4)$ & $8(0.7)$ & & $0.59^{*}$ \\
\hline HSIL (\%) & $1(0.1)$ & $2(0.3)$ & $2(0.2)$ & & $1^{*}$ \\
\hline Total (\%) & $670(100)$ & $741(100)$ & $1093(100)$ & & \\
\hline
\end{tabular}

Note:* Fisher exact probability method:

ASC-US: Atypical Squamous Cell of Uncertain Significance; LSIL: Low Grade Squamous Intraepithelial Lesion; HSIL: High Grade Squamous Intraepithelial Lesion.

\section{HPV Infection Status of Personnel in 2017}

\section{Relationship Between Age and HPV Infection and TCT Test Result}

The HPV infection rates of the age groups of 21 to 30 years,
31 to 50 years, and over 51 years were $9.6 \%$, 8.6\%,and $13.3 \%$, respectively. The infection rate and the cytological positive rate increased as the age increases.(Table 2 , Pearson $\chi 2=4.43, \mathrm{P}=0.11$, Table 1, Pearson $\chi 2=4.34, \mathrm{P}=0.11$ ).

Table 2: 2017 HPV Test Result by Age Group.

\begin{tabular}{|c|c|c|c|c|}
\hline \multirow{2}{*}{ Age Group } & \multicolumn{2}{|c|}{ HPV Resulta } & \multicolumn{2}{c|}{ TctResult } \\
\cline { 2 - 5 } & Negative (\%) & Positive(\%) & NILM (\%) & ASC-US and above (\%) \\
\hline $21 \sim 30$ & $246(90.4$ & $26(9.6)$ & $266(97.1)$ & $8(2.9)$ \\
\hline $31 \sim 50$ & $510(91.4)$ & $48(8.6)$ & $541(96.4)$ & $20(3.6)$ \\
\hline $51 \sim$ & $221(86.7)$ & $34(13.3)$ & $242(93.8)$ & $16(6.2)$ \\
\hline Total & $977(90.0)$ & $108(10.0)$ & $1049(96.0)$ & $44(4.0)$ \\
\hline
\end{tabular}

Note: a $\chi 2$ test, Pearson $\chi 2=4.43, \mathrm{P}=0.11$

b $\chi^{2}$ test, Pearson $\chi^{2}=4.34, P=0.11$

\section{Different Subtypes of HPV Infection}

Among the 108 HPV-positive medical staff, 82 cases (75.93\%) were single infection, and 26 cases were mixedInfection(24.07\%). The HPV subtypes were divided into high-risk type, potential highrisk type and low-risktype. The common subtypes of high-risk
HPV were HPV52, HPV58, HPV16, etc, the results showed that thenumber of infected people of high-risk HPV was 103 (73.04\%). The common potential high-risk HPV subtypes include HPV53, etc, it has a number of infected people of 26 (18.44\%). The common subtypes of low-risk HPVincludes HPV81, and the results showed that the number of infected people was 12 (8.51\%) (see Table 3).

Table 3: 2017 HPV Infection Status of Screening Personnel in a First-class Hospital at Grade 3 inShanghai.

\begin{tabular}{|c|c|c|c|}
\hline HPV Subtype & & \# of People & Infection Rate (\%) \\
\hline \multirow{4}{*}{ High risk subtype } & HPV52 & 31 & 21.99 \\
\hline & HPV58 & 15 & 10.64 \\
\hline & HPV16 & 13 & 9.22 \\
\hline & $\begin{array}{c}\text { others (HPV56/59/39/68/18/33/31/51 } \\
\text { /35/45) }\end{array}$ & $44(8 / 8 / 7 / 7 / 4 / 4 / 2 / 2 / 1 / 1)$ & 31.21 \\
\hline \multirow{5}{*}{ Potential high-risk subtype } & HPV53 & 19 & 13.48 \\
\hline & HPV66 & 2 & 1.42 \\
\hline & HPV73 & 2 & 1.42 \\
\hline & HPV82 & 2 & 1.42 \\
\hline & HPV26 & 1 & 0.71 \\
\hline \multirow{3}{*}{ Low risk subtype } & HPV81 & 7 & 4.96 \\
\hline & HPV6 & 3 & 2.13 \\
\hline & HPV11 & 2 & 1.42 \\
\hline
\end{tabular}


Relationship Between Different Screenings Srategies and Cervical Biopsy

Using the positive results of the 2017 biopsy (LSIL or HSIL lesions) as criteria, the TCT and HPV test result from2015 to 2017 were analyzed. 3 cases of negative result were missed diagnosed $(8.1 \%)$ in the subjects whencytology was used as single screening strategy and all of them were LSIL lesions. While using HPV as singlescreening strategy, 9 patients were diagnosed as positive (19.1\%), including 7 with LSIL and 2 patients withHISL. 6 patients with HPV-negative result was missed diagnosed (31.6\%), all of which were LSIL lesions. HPV16,the most relevant subtype with cervical cancer, has a high positive biopsy coincidence rate of $60 \%(3 / 5)$. Dueto the small number of biopsies, the relevance of screening is not statistically significant. The second test withhigher positive rate of biopsy was single cytology test. The biopsy rate of cytology was $41.4 \%$, with 10 cases ofLSIL and 2 cases of HSIL. The positive rate for both TCT and HPV test was 35\% (7/20), while the positive biopsy rate for at least one of the two tests was $25 \%$ (14/56). The missed diagnosis rate was 29.7\% (11/37) for those patients with negative CTC test result in 2015 and 2016 (Table 4).

Table 4: Histological biopsy compliance rate for different screening strategies.

\begin{tabular}{|c|c|c|c|c|}
\hline Screening Stratergy & NILM(\%) & LSIL(\%) & HSIL(\%) & Total \\
\hline Single TCT negative & $34(91.9)$ & $3(8.1)$ & $0(0.0)$ & $2(6.9)$ \\
\hline Single TCT positive & $17(58.6)$ & $10(34.5)$ & $0(0.0)$ & 19 \\
\hline HPV negative & $13(68.4)$ & $6(31.6)$ & $2(4.3)$ & 47 \\
\hline HPV positive & $38(80.9)$ & $7(14.9)$ & $2(10.0)$ & 5 \\
\hline HPV16 positive & $2(40.0)$ & $5(25.0)$ & $2(3.6)$ & 20 \\
\hline TCT and HPV positive & $13(65.0)$ & $12(21.4)$ & $2(5.4)$ & 56 \\
\hline TCT or HPV positive & $42(75.0)$ & $9(24.3)$ & & 37 \\
\hline Previous TCT negative & $26(70.3)$ & & & \\
\hline
\end{tabular}

\section{Risk Analysis of Positive Test Results}

Dividing the histological biopsy results into 2 groups, negative and positive (including LSIL and HSIL), we thenuse them as dependent variable of the logistic regression model along with ASC-US negative samples. Aftercorrecting age-related factors, the analysis was designed to study the relationship between dependent variableswith TCT results (negative and positive), HPV results (negative and positive), HPV type 16 results (negative andpositive), and the TCT results (negative and positive) for the first 2 year. The TCT results and HPV16 infectionresults were correlated with histological results, with an odds ratio (OR) of 7.99 (95\% CI: 1.97-32.36) ( $\mathrm{P}=0.004)$ and of 10.3 (95\% CI: 1.24-85.15) $(\mathrm{P}=0.031$ ), respectively (Table 5 ). The Odds ratio becomes $7.87 \%$ (95\% CI:1.85-33.54) ( $\mathrm{P}=0.005)$ for the TCT result after adding Cytological and HPV 16 results as the two independentvariables while the correlation between HPV 16 infection and biopsy results disappeared $(\mathrm{P}=0.06)$ (Tables 5 \& 6).

Table 5: Single Logistic Regression Analysis of Biopsy Results of Screening Personnel in a First-class Hospital at Grade 3 in Shanghai by Risk Factors.

\begin{tabular}{|c|c|c|c|c|c|c|}
\hline \multirow{2}{*}{ Factors } & \multirow{2}{*}{$\beta$} & \multirow{2}{*}{$\mathbf{x}^{2}$} & \multirow{2}{*}{ P Ratio } & \multirow{2}{*}{ Ratio } & \multicolumn{2}{|c|}{ 95\% Confidence Interval } \\
\hline & & & & & Lower limit & Upper limit \\
\hline TCT positive & 2.078 & 8.468 & 0.004 & 7.985 & 1.97 & 32.36 \\
\hline HPV positive & -0.652 & 1.1 & 0.294 & 0.521 & 0.154 & 1.761 \\
\hline HPV16 positive & 2.328 & 4.647 & 0.031 & 10.256 & 1.235 & 85.151 \\
\hline $\begin{array}{l}\text { TCT positive in } \\
\text { previous } 2 \text { years }\end{array}$ & 0.125 & 0.009 & 0.923 & 1.134 & 0.09 & 14.21 \\
\hline
\end{tabular}

Table 6: Multiple Logistic Regression Analysis of Biopsy Results of Screening Personnel in a First-class Hospital at Grade 3 in Shanghai by Risk Factors.

\begin{tabular}{|c|c|c|c|c|c|c|}
\hline \multirow{2}{*}{ Variable } & \multirow{2}{*}{$\beta$} & \multirow{2}{*}{$x^{2}$} & \multirow{2}{*}{$\mathbf{P}$} & \multirow{2}{*}{ Ratio } & \multicolumn{2}{|c|}{$95 \%$ Confidence Interval } \\
\hline & & & & & Lower limit & Upper limit \\
\hline Age & 0.035 & 1.632 & 0.201 & 1.035 & 0.982 & 1.092 \\
\hline TCT result & 2.063 & 7.773 & 0.005 & 7.867 & 1.845 & 33.54 \\
\hline HPV16 result & 2.331 & 3.551 & 0.060 & 10.288 & 0.911 & 116.219 \\
\hline Constant & -6.365 & 11.175 & 0.001 & 0.002 & & \\
\hline
\end{tabular}




\section{Conclusion}

The HPV infection rate of the respondents in this study was $10.0 \%$, while there have been many studies aboutthe correlation between HPV infection and cervical cancer and precancerous lesions. The results of thosestudies vary as the region and test subject changes. According to the HPV test result of a First-class Hospital atGrade 3 in Hunan, the HPV infection rate of female who visited STD clinic and Urology clinic was $45.33 \%$. Thepeak age of infection was 26-35 years old, but the difference in infection rates among different age groups wasnot statistically significant [10]. The HPV infection rate from the medical examination of women in a First-classHospital at Grade 3 in Beijing was $12.84 \%$. According to the result, infection rate was the highest among womenunder 26 years old and decreases as age grows [11]. The HPV positive rate of women under 39 years old inShenzhen was significantly higher than those of 40 to 49 -year-old and of over 50 years old [12]. In Laos,percentage of HPV infection for women in age of $30-54$ years old is $47.7 \%$ [13]. The HPV infection rate ofmedical staff in our study was $10.84 \%$ and was lower than that of hospital specialist outpatients and generalmedical examination. The author believes that it may be related to the target population of this study. Medicalstaff is a special occupational group with has higher awareness of HPV infection than the general populationand more medical expertise. They pay more attention to personal protection in daily life and have better diseasedetection as well as treatment. These can all be some of the reasons why the HPV infection rate is lower for themedical staff than the general population.

On the other hand, the HPV subtypes of infection in this study vary a lot. In this study, the most popular infectionsubtype is HPV52, 53,58 and 16. While in the past, most researches are concentrated in types of HPV16, 52, 58and other high-risk subtypes [14,15]. HPV16 is classified as a high-risk HPV and have been confirmed by studiesthat its persistent infection is significantly associated with advanced cervical cancer [16]. Same correlation wasfound in our study. However, the correlation disappears in multiple logistic regression analysis. The possiblereasons for that could be the limitation of number of samples or TCT test positive rate was higher than that of HPV test.

The rise of HPV testing has led to debates about whether HPV testing can replace cytology as a first-linescreening method for cervical lesions. The results of this study suggest that the cytology test result isindependent risk factors for cervical lesions, and its specificity and sensitivity of cytological detection is higherthan HPV detection. The positive rate of biopsy positive for both cytology and HPV is not higher than that ofcytology screening alone, thus this study supports cytology as a first-line screening tool. However, theeffectiveness of cytology highly depends on qualified control and the cytological quality of our hospital is withinthe range of reference values published by the American Association of Pathologists (CAP) [17]. Since thepreviously reported ASC-US rate is at the lower limit of the CAP range, the ASC-US rate in the 2017 results hasincreased significantly from the previous two years and is conducive to improving cytological sensitivity. Thereis a controversy of the reasonable screening cycle. According to this study, a 1-year screening cycle is necessaryas if cytology is used as a single screening method, there is still a $29.7 \%$ or more of the risks of LSIL given anegative cytology result for the previous 2 years. HPV typing detection, especially HPV16 and 18, has obviousadvantages over untyped HPV testing by improving the accuracy of positive prediction. This is also the reasonfor the immediate referral of colposcopy in HPV16 and/or HPV18 positive in the American Society ofColposcopy and Cervical Pathology (ASCCP) guidelines.

This study was carried out in a group of medical staff in a Firstclass Hospital at Grade 3 in Shanghai. It analyzedthe surveyed population's HPV infection rate and HPV infection subtypes and analyzed the positive predictioneffects of HPV detection and cytology screening. Due to the small sample number, no correlation with histologywas found except for HPV16 in this study. Therefore, in future research, we can expand the sample size of thesurvey population, conduct research on the correlation between the highly pathogenic HPV type and histology,and even explore the highly pathogenic HPV type of the Chinese population for further independent detection.That will effectively increase the specificity of HPV as a screening for cervical lesions. This providesindependent test a significant meaning especially while using as primary screening in remote areas with scarcecytological diagnostics by reducing medical costs and improving screening rate.

\section{Conflict of Interest}

The author declares that this article has no actual or potential conflict of interest.

\section{References}

1. (2018) World Health Organization / Cancer Fact: Cervical uteri Source Globocan 2018 [EB/OL].

2. Ebisch RM, Siebers AG, Bosgraaf RP, Leon Fag Massuger, RuudLm Bekkers, et al. (2016) Triage of high-risk HPV positive women in cervical cancerscreening [J]. Expert Rev Anticancer Ther16(10): 1073-1085.

3. Austin RM (2010) Exhortations to abandon the Pap test as a routine initial cervical screening test are stillpremature and carry significant risks[J]. Diagn Cytopathol38(11): 783-787.

4. (2016) The American College of Obstetricians and Gynecologists. Cervical Cancer Screening and Prevention [J].ObstetGynecol 127(1): e1e20.

5. Shiqi Hu, Yujing Yang, Xia Zhao (2018) Value of HPV test, liquid-based cytologic test (TCT) and combination ofthe two methods in screening cervical disease 34(5): 371-376.

6. Long W, Yang Z, Li X, Ming Chen, Jie Liu, et al. (2018) HPV-16, HPV-58, and HPV-33 are the most carcinogenic HPV genotypes inSouthwestern China and their viral loads are associated with severity of premalignant lesions in the cervix[J]. Virol J15(1): 94.

7. Li M, Du X, Lu M, Weiyi Zhang, Zhihui Sun, et al. (2018) Prevalence characteristics of single and multiple HPV infections in women withcervical cancer and precancerous lesions in Beijing, China[J]. J Med Virol, 
8. Pan Q J, Hu SY, Guo HQ, Wen-Hua Zhang, Xun Zhang, et al. (2014) Liquid-based cytology and human papillomavirus testing: a pooled analysisusing the data from 13 population-based cervical cancer screening studies from China[J]. Gynecol Oncol 133(2): 172-179.

9. Stoler M, Kim KR, Bergeron C, et al. (2014) Squamous cel l tumours and precursors, in Kurmam RJ, Carcangiu ML,Herrington CS, et al. WHO Classification of Tumours of Female Reproductive Organs, $4^{\text {th }}(\mathrm{Edn}$,$) .$ IARC, Lyon: pp: 172-182.

10. Miao Liu, Jianrong Ye, Shishan Yuan (2018) Gene Types Analysis of human papillomavirus in out-patients andhealthy cases15(2): 39-41.

11. Limin Zhang, Fang Fang, Kelin Chen (2018) Detection and analysis on gene subtypes of cervical human papillomavirus infection in women with pregnancy and physical examination 22(2): 265-268.

12. Jinjin Xu, Yuxia Zhang (2017) Screening and prevention strategies of two cancers of suitable age women in Guangming New District of Shenzhen City from 2011 to 201532(10): 2168-2171.

ISSN: $2574-1241$

DOI: 10.26717/BJSTR.2020.27.004482

Hu Min-Min. Biomed J Sci \& Tech Res

(C) This work is licensed under Creative

Submission Link: https://biomedres.us/submit-manuscript.php
13. Takamatsu R, Nabandith V, Pholsena V, Phouthasone Mounthisone, Katsu Nakasone, et al. (2017) Cervical cytology and human papillomavirus amongasymptomatic healthy volunteers in Vientiane, Lao PDR[J]. BMC Cancer17(1): 872

14. Jiayan Li, Yuwu Luo, Chao Bi (2014) Analysis of 1786 cases of HPV genetic subtype 28(6): 595-597.

15. Zheng HS, Zheng DN, Li JM, et al. (2017)The survey of human papillomavirus infection and subtype in 2663 women [J]. Contemporary Medicine17(21): 20-21.

16. KarbalaieNiya MH, Keyvani H, SafarnezhadTameshkel F, Mostafa SalehiVaziri, SedighehTeaghinezhad-S,et al. (2018) Human Papillomavirus Type 16 IntegrationAnalysis by Real-time PCR Assay in Associated Cancers[J]. Transl Oncol11(3): 593-598.

17. Tao X, Austin RM, Zhang H, et al. (2015) Pap Test Reporting Rates for Conventional Smear and Liquid-BasedCervical Cytology from the Largest Academic Women's Hospital in China: Analysis of 1,248,785 Pap Test Reports [J]. Acta Cytol 59(6): 445-451.

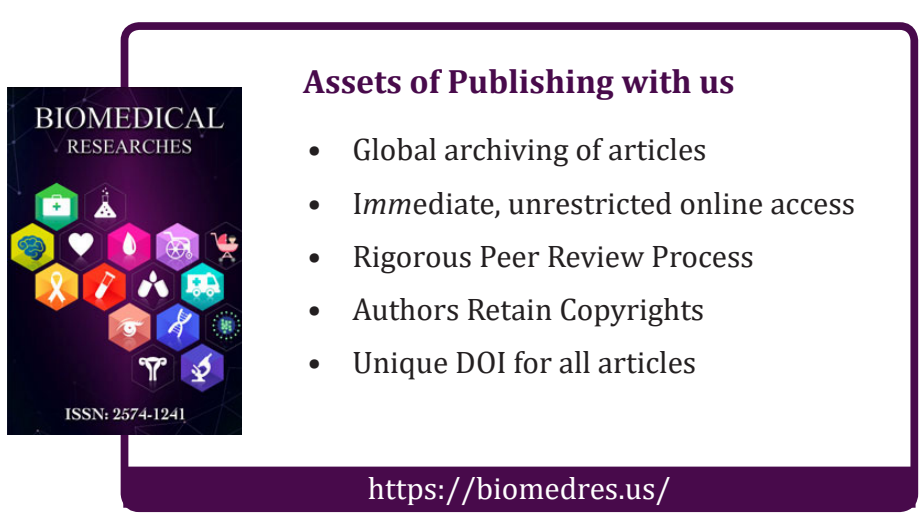

